\title{
Survey of large-amplitude flapping motions in the midtail current sheet
}

\author{
V. A. Sergeev ${ }^{1}$, D. A. Sormakov ${ }^{1}$, S. V. Apatenkov ${ }^{1}$, W. Baumjohann ${ }^{2}$, R. Nakamura ${ }^{2}$, A. V. Runov ${ }^{2}$, T. Mukai ${ }^{3}$, and \\ T. Nagai ${ }^{4}$ \\ ${ }^{1}$ St. Petersburg State University, Petrodvoretz, 198504 St. Petersburg, Russia \\ ${ }^{2}$ Austrian Academy Science, Space Research Inst., Schmiedlstrasse 6, Graz, 8042, Austria \\ ${ }^{3}$ ISAS, Sagamihara, Kanagawa 229, Japan \\ ${ }^{4}$ Tokyo Inst Technology., Dept Earth \& Planetary Sciences., Ookayama 2-12-1 Meguro., Tokyo, 152-8551, Japan
}

Received: 27 February 2006 - Revised: 10 May 2006 - Accepted: 14 June 2006 - Published: 9 August 2006

\begin{abstract}
We surveyed fast current sheet crossings (flapping motions) over the distance range $10-30 R_{E}$ in the magnetotail covered by the Geotail spacecraft. Since the local tilts of these dynamic sheets are large and variable in these events, we compare three different methods of evaluating current sheet normals using 4-s/c Cluster data and define the success criteria for the single-spacecraft-based method (MVA) to obtain the reliable results. Then, after identifying more than $\sim$ 1100 fast CS crossings over a 3-year period of Geotail observations in 1997-1999, we address their parameters, spatial distribution and activity dependence. We confirm that over the entire distance covered and LT bins, fast crossings have considerable tilts in the YZ plane (from estimated MVA normals) which show a preferential appearance of one (YZ kinklike) mode that is responsible for these severe current sheet perturbations. Their occurrence is highly inhomogeneous; it sharply increases with radial distance and has a peak in the tail center (with some duskward shift), resembling the occurrence of the BBFs, although there is no one-to-one local correspondence between these two phenomena. The crossing durations typically spread around 1 min and decrease significantly where the high-speed flows are registered. Based on an $\mathrm{AE}$ index superposed epoch study, the flapping motions prefer to appear during the substorm expansion phase, although a considerable number of events without any electrojet and auroral activity were also observed. We also present statistical distributions of other parameters and briefly discuss what could be possible mechanisms to generate the flapping motions.
\end{abstract}

Keywords. Magnetospheric physics (Magnetotail; MHD waves and instabilities; Plasma sheet)

Correspondence to: V. A. Sergeev

(victor@geo.phys.spbu.ru)

\section{Introduction}

Magnetospheric (magnetopause and magnetotail) current sheets are among those rare space current sheets which are available for direct observations. Their dynamics provide important clues to understand the plasma transport and conditions for explosive tail instability (substorm) to occur. One dynamic phenomenon, the flapping motions of the tail current sheet, manifests in observations as dramatic variations of the main magnetic field component (tail-aligned, $B_{x}$ ) in the tail, often with the change in the $B_{x}$ sign, indicating a crossing of tail current central surface. Being known since first spacecraft measurements in the tail (Speiser and Ness, 1967), it is frequently observed in the active plasma sheet and is interesting both as a spectacular phenomenon (origin, properties, possibility to use as a diagnostic tool of active processes), as well as a tool to probe the sheet structure by crossing quickly across considerable portions of the current sheet (CS) thickness. There was a number of past studies which noticed their association with interplanetary shocks (McComas et al., 1986), with substorms and/or fast flows (Toichi and Miyazaki, 1976, Bauer et al., 1995, Sergeev et al., 1998), although counter-examples were also provided. The ideas about their characters included solar wind blowinduced surface waves in the X-direction (e.g. Fairfield et al., 1981), or kink-like oscillations in the $Y$ direction (e.g. Lui et al., 1978; Nakagawa and Nishida, 1989), however, the difficulties of separating temporal and spatial variations, and of measuring the gradients and current sheet tilts strongly limited the possibilities to study these phenomena with a single spacecraft.

The situation drastically changed recently after the launch of the four-spacecraft Cluster which allows one to probe gradients and to better investigate both the current sheet structure and its dynamics. Already first event studies (starting from Zhang et al., 2002) indicated very large and variable tilts in the $\mathrm{YZ}$ plane and a predominant propagation

Published by Copernicus GmbH on behalf of the European Geosciences Union. 
in the Y-direction associated with flapping motions, and this was thereafter confirmed in statistical studies (Sergeev et al., 2004; Runov et al., 2005) which allowed one to identify them as the "kink-like" perturbations. However, the polar orbit of Cluster strongly limits the amount of current sheet crossings recorded, as well as their distance in the tail (they were all clustered near Cluster apogee at $r$ around $18 \mathrm{Re}$ during the first years of operation). To address the occurrence in the tail, possible changes in the perturbation mode and characteristics with distance (both along and across the tail), as well as activity dependence and some other aspects which require one to analyze the large amount of data, one has to include other data sources. In this paper we use the Cluster experience and the extensive observations in near-equatorial tail region made by Geotail to study the flapping motions statistically.

The paper is organized as follows. In the next section we compare 3 different techniques available at Cluster for current sheet normal determination with the purpose of defining the practical criterion to use a single-spacecraft-based Minimal Variance Analysis technique when surveying the Geotail data. Then in the main Sect. 3 we analyse the Geotail observations, and thereafter discuss the results in Sect. 4.

\section{Tilt characterization during fast current sheet cross- ings: Cluster results}

Some results and conclusions of the flapping survey presented below depend on the accuracy of estimated local current sheet (CS) tilts, which requires some comments and an additional study. No perfect method exists to probe the sheet tilt and structure, even with data obtained at a few regularly spaced observation points, like the four Cluster spacecraft. The advantage of Cluster is that three different methods could be used, which, fortunately, depend on different assumptions, so their cross-comparison helps to be sure in the correctness of the interpretation results. The four spacecraft timing method, which assumes a planar sheet structure and either a constant speed, or a constant thickness (see, e.g. Haaland et al., 2004), gives both the sheet orientation and the normal component of its propagation velocity. Also, using the magnetic field gradient linear estimation technique (Chanteur et al., 1998), the sheet tilt can be determined based on the direction of the estimated electric current $(j$, obtained in the center of 1-D sheet, planar sheet structure is also assumed). This vector is suggested to lay on the neutral sheet plane, therefore, the unit vector of the sheet normal can be obtained as $\mathbf{n}=[\mathbf{l} \times \mathbf{j}] /[[\mathbf{l} \times \mathbf{j}] \mid$, where $\mathbf{l}$ is the maximum B-variability direction obtained from MVA (Runov et al., 2005). The agreement between these methods can be used as an indication that we deal with nearly a 1-D current sheet which conserves its orientation during the CS crossing. Finally, the well-known Minimum Variance Analysis (Sonnerup and Schneible, 1998) was widely used for many years to estimate the sheet orientation, although, in a strict sense, no physical law guarantees that it provides the accurate estimate even in a nearly 1-D current sheet. For example, in the Harris-type 1-D sheet the magnetic variance is zero not only in the normal direction, but also along the direction of electric current, so some other factors, either field-aligned currents or perturbations, will determine what will be the smallest MVA variance direction.

Previous Cluster-based studies of solar wind discontinuities (based on a comparison between timing and MVA methods) indicated that it is possible to obtain the success criteria for the MVA to work. Particularly, for solar wind discontinuities Knetter et al. (2004) found the good accuracy of MVA (with sufficient amount of measurement points in the discontinuity) when the magnetic shear was large and the medium-to-minimal eigenvalues ratio $r_{23}=\lambda_{2} / \lambda_{3}$ was large ( $>8$ in their case). Unfortunately, these practical criteria cannot be easily transferred to another object (to the tail current sheet, in our case), where the underlying physics can be different. Also, with the increasing threshold of $r_{23}$, the amount of usable data strongly decreases, so some trade off between the high accuracy and sufficient amount of remaining events is unavoidable. This is what we investigate in this section based on Cluster data, and what will then be used later on in the analysis of Geotail data.

We used the Cluster FGM observations at 1-s resolution during July-October 2001 and 2004 tail seasons, in which the spacecraft tetrahedron has comparable sizes $(\sim 1000$ $1500 \mathrm{~km})$. Short $(30 \mathrm{~s}<\Delta t<300 \mathrm{~s})$ crossings of the current sheet with considerable $B_{x}$ change (being a sizable fraction of the lobe field $\mathrm{BL}$, that is $\Delta B_{x}>0.5 \mathrm{BL} \sim 15 \mathrm{nT}$ ) have been identified, which resulted in 186 (and 187) crossings identified in years 2001 (and 2004). A subset of $\sim 200$ crossings was then selected in which all four spacecraft crossed (or touched) the neutral sheet, where a sufficient amount of curlometer determinations were accurate enough ( $\mid$ Div $B|/| C \operatorname{CurlB} \mid<0.3$ in $>60 \%$ of points) and variations at all SC have similar shapes (allowing the use of timing); see Runov et al. (2005) for the description of the selection procedure and events in 2001, as well as all data procession details.

Cross-comparison of current sheet normals obtained by different methods in the later analysis is based on scalar products between the pairs of normals from which the angular difference between the two normals $(\Delta \alpha)$ was obtained. $\mathrm{Cu}-$ mulative probability distribution functions (PDF) in Fig. 1 (for events in which the normals could be obtained by both compared methods, notice that amounts of events differ in different pairs) shows that the best agreement is observed between timing (TIM) and current-based (CUR) methods. For them more than $2 / 3$ of all events agree to within a $\Delta \alpha=15^{\circ}$ difference. Agreement of these two methods with the MVA determinations is not as good, requiring a much broader interval $\Delta \alpha<35^{\circ}$ for $2 / 3$ of all events to agree. No selection on any other parameter/condition characterizing MVA performance (e.g. dependence on the ratio $r_{23}$ ) was used at this step; this is what we explore next. 


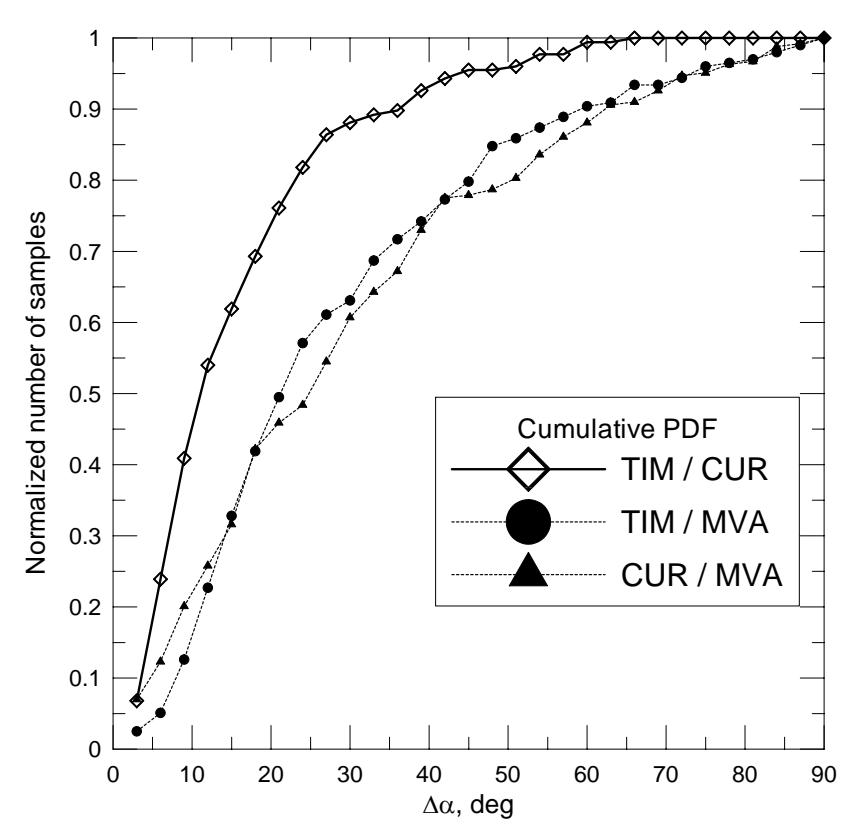

Fig. 1. Cumulative probability distribution function of events with angular difference between two normals (obtained with TIM, CUR or MVA methods) below the given $\Delta \alpha$ angular difference.

We choose then a subset of 109 crossings in which the TIM/CUR agreement is good $\left(\Delta \alpha<15^{\circ}\right)$. Using the TIM normals as our guess of the true normal in these cases, we used MVA determinations from each of the 4 spacecraft to compare their angular deviations from true normal as a function of $r_{23}$ (in total having 428 intervals). Figure 2 indicates that indeed the angular deviation is small for large $r_{23}$ values, and grows with a decrease of this ratio; similar to the results by Knetter et al. (2004) the error curve shows a considerable change around the $r_{23}=8$ threshold. However, the amount of events strongly decreases when the $r_{23}$ decreases from 20 until 3, so for practical purposes one should compromise between the requirements, to obtain a good accuracy and to keep a large enough and representative data set for doing statistics. With the threshold $r_{23}>4$ adopted below for our statistical study of Geotail data, most of normal determinations are expected to be accurate to within $20-30^{\circ}$.

\section{Geotail observations of flapping motions}

The present Geotail equatorial orbit provides an excellent opportunity to survey the plasma sheet dynamics between 10 and 30 Re since 1995. We used magnetic field (MGF) and plasma (LEP) parameters at a 12-s resolution to select and characterize the events and a 3-s magnetic field data to perform the MVA. We surveyed the spatial region $X<8$, $|Y|<15$ Re GSM and excluded magnetosheath samples in the near flank regions. We defined fast CS crossings by the requirement of a fast ( $30 \mathrm{~s}<\Delta t<300 \mathrm{~s})$, monotonous change in

\section{MVA accuracy depending on eigenvalue ratio $\left(r_{23}\right)$}

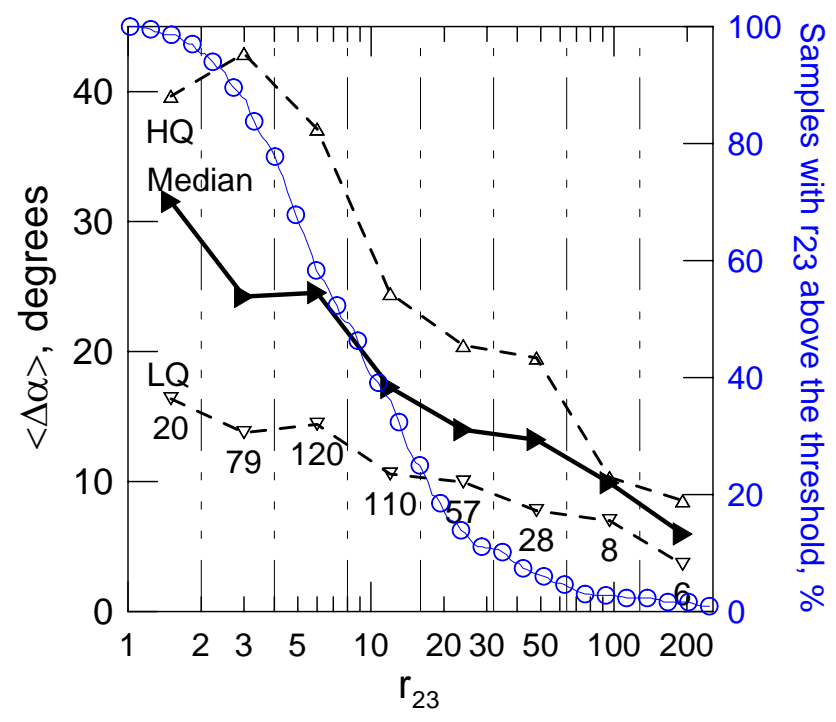

Fig. 2. Performance of MVA as a function of eigenvalue ratio $r_{23}$. Angular differences from the TIM normal (characterized by median and high (HQ) and low (LQ) quartiles, where the number of events in every bin) is shown together with the number of events (circled curve) with $r_{23}$ exceeding the given threshold.

the $B_{x}$-component of considerable amplitude $\left(\left|\Delta B_{x}\right|>B L\right.$, where $B L$ is the equivalent lobe field obtained from the pressure balance as $\left.B L=\left(B^{2}+2 \mu_{0} n T_{i}\right)^{1 / 2}\right)$. With these criterions we identified 1270 fast current sheet crossing events during $~ 3$ years (December 1996-May 1999) to be analysed. Their spatial coverage is shown in Fig. 3.

\subsection{Occurrence and current sheet parameters}

Characteristic values of plasma velocity $\left(V_{x}\right.$ and $\left.V_{y}\right)$ and magnetic field components in the neutral sheet $\left(B_{x} \sim 0\right)$ are given in Fig. 4. The magnetic field magnitude in the CS center is nonzero but most frequently small ( $B_{y z}$ median is about 2nT). The $B_{y}$ component has symmetric distribution, whereas $B_{z}$ is shifted towards positive values, reflecting the closed character of the field lines in the majority of the surveyed events. Unlike the narrow Gaussian $V_{y}$ distribution, $V_{x}$ has a wide non-Gaussian distribution. It has a large amount $(>25 \%)$ of low-speed events $\left(V_{x}<100 \mathrm{~km} / \mathrm{s}\right)$, but also displays pronounced high-speed flow wings for both earthward and tailward flows, showing that many fast crossings had associated fast plasma flows (BBFs).

A large amount of high speed flows is also seen in the top charts of Fig. 5, however, the average values (see Ydependent plot) are near $400 \mathrm{~km} / \mathrm{s}$, which is the conventional threshold for the BBFs (Angelopoulos et al., 1994). Y-plot shows somewhat higher flow speed in the middle-dusk sector of the tail, where the average velocity strongly increases 


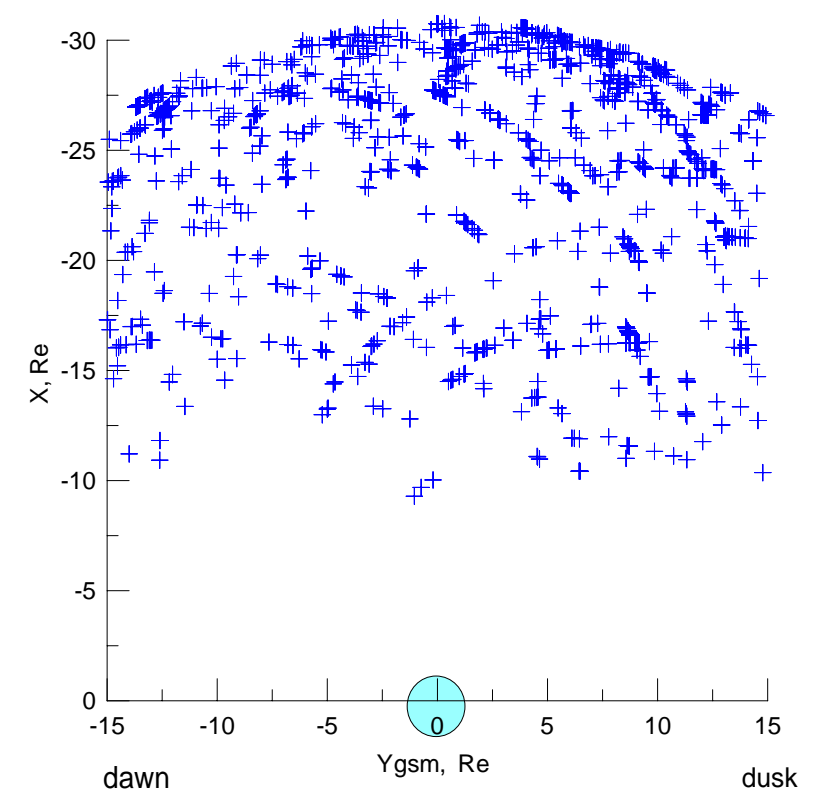

Fig. 3. Spatial distribution of fast current sheet crossings in the equatorial plane.

with the distance from $\sim 200 \mathrm{~km} / \mathrm{s}$ in the $10-15$ Re bin towards $>400 \mathrm{~km} / \mathrm{s}$ in the $25-30 \mathrm{Re}$ bin, consistent with previous flow speed statistics in the earthward flux transfer events (e.g. Schödel et al., 2001).

We also computed the occurrence of crossings and compare it with the occurrence of BBFs. (These are different kinds of statistics: the flapping occurrence is a statistic of the events with different durations, whereas the BBF occurrence is s statistic of 12-s samples with plasma $\beta>1$ and a flow velocity above the $400-\mathrm{km} / \mathrm{s}$ threshold. To have the occurrences in comparable quantities we normalized the amount of flapping events by the amount of minutes of observations with $\beta>1$, after noticing that the average duration of the flapping event is close to one minute.) As seen at the bottom plots of Fig. 5, the occurrence of crossings is very inhomogeneous over the region studied, and this is probably the most important new result of our survey. It increases considerably with the distance, and maximizes in the central sector of the tail (with some duskward shift). The significance of this inhomogeneity is further emphasized by a comparison to the occurrence of the fast plasma flows (BBFs, red traces) which shows similar behavior. This suggests a close relationship between these two phenomena, which will be further discussed in Section 4 . The occurrence of crossings is roughly a factor 20 smaller than the occurrence of BBFs. (We shall not later discuss thoroughly the different Y-positions of the peak in flapping and BBF distributions, because we found that the shape of BBF Y-distribution varies with the varying selection criteria. In particular, lifting the $\beta>1$ condition results in the dusk-shifted distribution which matches the flapping occurrence.)
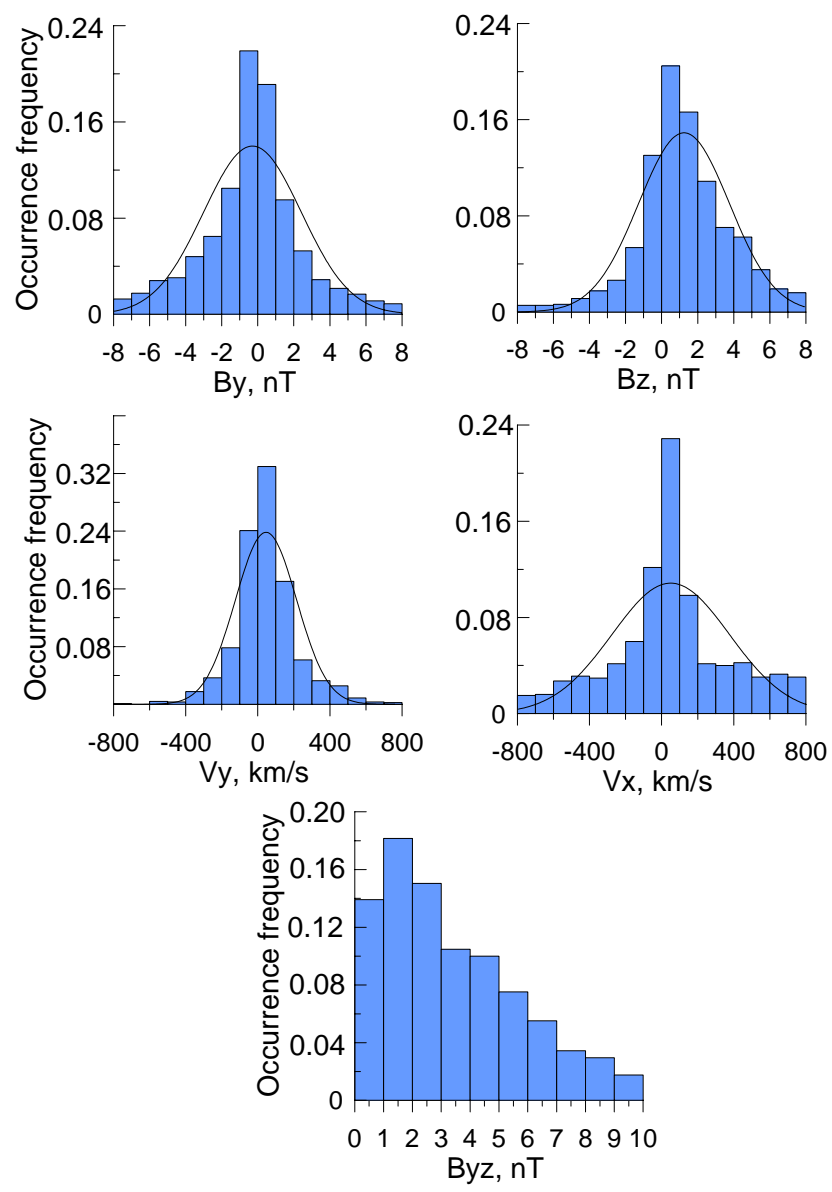

Fig. 4. Normalized occurrence frequency distributions of magnetic field and plasma flow velocity components in the neutral sheet during fast plasma sheet crossings (their Gaussian fits are also shown). $\left.B_{y z}=\left(B y^{2}+B z^{2}\right)^{1 / 2}\right)$ is the magnetic field in the neutral sheet.

The duration of the crossings ( $\mathrm{dT}$ is the time between observations of $\min / \max B_{x}$ values during the CS crossing) is, on average, about 1 min (see dT medians in Fig. 5); it shows some tendency to decrease with distance, displaying some anticorrelation with the average velocity behavior. This relationship is directly seen in Fig. 6, showing the drastic difference between very short crossings seen in the high speed flows as contrasted to much slower (with large spread in duration) crossings in the low speed plasma.

\subsection{Characteristics of current sheet in the flapping wave}

Now we investigate the properties of the perturbations causing the fast current sheet crossings. The first characteristic of importance is the tilt of the flapping sheets available from the MVA techniques, whose accuracy was discussed in Section 2. After obtaining the MVA estimates based on 3-s time resolution data (with a requirement that at least 15 data points during the crossing should be available for the MVA analysis) we studied statistics and selected the eigenvalue 

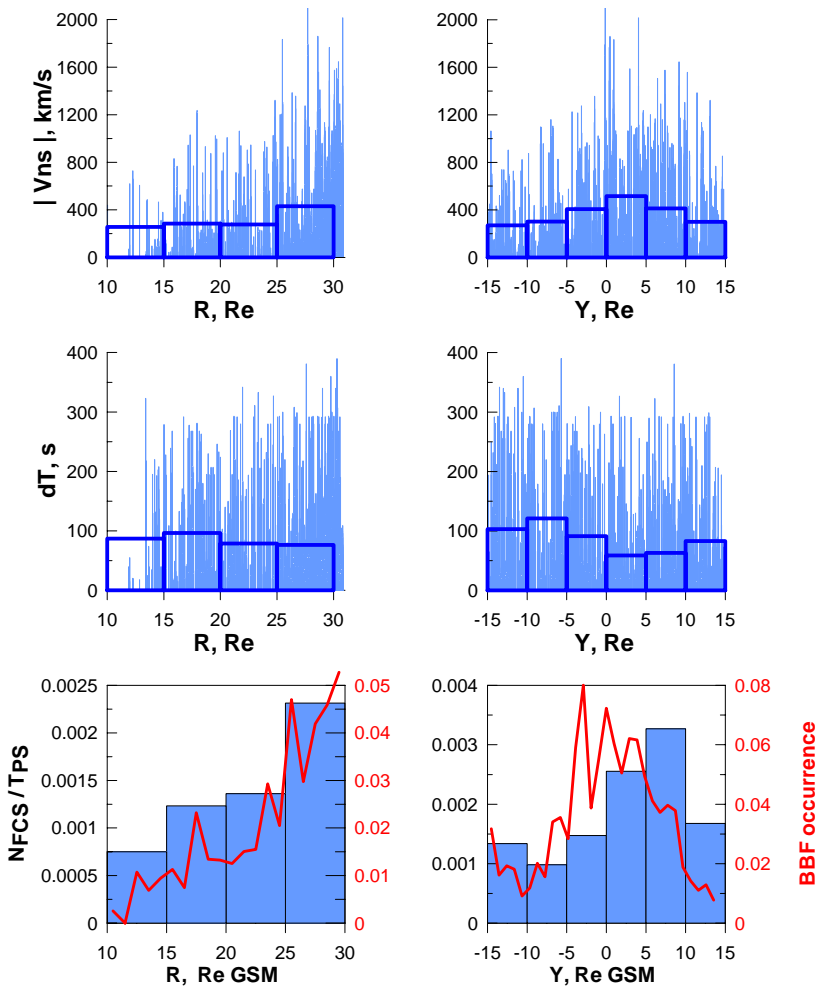

Fig. 5. Dependence of neutral sheet flows, duration of CS crossing and occurrence frequency of flapping events against the distance from the Earth $\left(R=\left(x^{2}+y^{2}\right) 1 / 2\right)$ and across the tail (Ygsm). The flapping occurrence is normalized to a total number of minutes of observation spent by the spacecraft in that spatial bin. Occurrence rate of fast plasma flows (with $|V x|>400 \mathrm{~km} / \mathrm{s}$ ) is shown for comparison in the two bottom panels.

ratio low threshold to be $r_{23}=4$. These requirements leave us 469 crossings (less than half of all events, but still a good number to study the spatial variation of characteristics). Since seasonal/daily tilting and warping of the current sheet produce large deviations of the neutral sheet normal from the Zgsm axis, for each event we computed the local normal expected for the given epoch at the given location, according to empirical neutral sheet shape given by Tsyganenko and Fairfield (2004), and rotated the obtained MVA normal (vector $\mathbf{n}_{3}$ ) to this local neutral sheet coordinate system - see Fig. 7a.

The distributions of the tilts in all spatial bins of Fig. 7 are surprisingly similar, lying near the unit circle in the $\mathrm{YZ}$ plane (means, $\left|n_{x}\right| \ll\left|n_{y}\right|,\left|n_{z}\right|$ since $n^{2}=1$ ). According to Fig. 2, under the threshold used, the expected MVA accuracy lies to within a $20-30^{\circ}$ deviation from the actual normal, therefore, the large deviations from the nominal normal are a real property of flapping motions in all spatial bins which have enough data points. The points are nearly homogeneously distributed along the unit circle, which means that strong deviations from the nominal normal (Z-axis) are a rule for the flapping current sheets. The ratio N1/N2 is used to character-

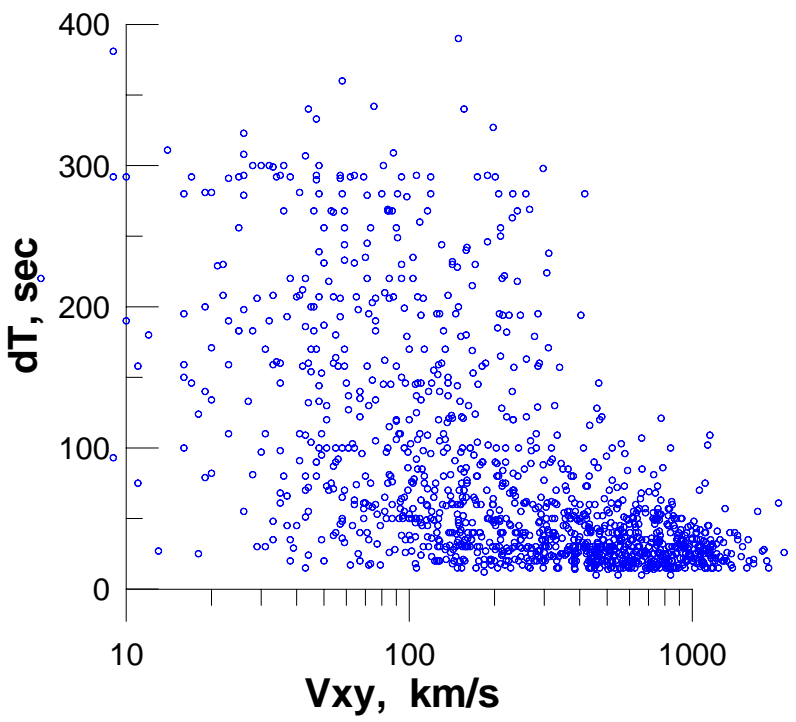

Fig. 6. Relationship between the neutral sheet flow amplitude $V x y=\left(\left(V x^{2}+V y^{2}\right)^{1 / 2}\right)$ and duration of the CS crossings. Short crossings (12 to $30 \mathrm{~s}$ ) were also included in this plot.

ize the amount of sheets N1 with normals close to the nominal normal (along $\mathrm{Z}$ ), as compared to the amount of anomalously tilted sheets N2 (see a scheme in the Fig. 7a). Comparing different bines we easily see that while in the central sector (bins a2, a3, b2, b3 - see a binning scheme in Fig. 7b) the normals near the nominal direction are more frequent $(\mathrm{N} 1 / \mathrm{N} 2 \gg 1)$, while near the flanks $(\mathrm{a} 1, \mathrm{a} 4, \mathrm{~b} 1, \mathrm{~b} 4)$ the anomalously tilted normals prevail $(\mathrm{N} 1 / \mathrm{N} 2 \leq 1)$.

The data base of fast current sheet crossings includes both isolated crossings, as well as sequences of crossings. We defined as a sequence a number (at least three) of sequential fast CS crossings, if the time difference between them does not exceed 300 s. Fifty-one sequences were identified with these criteria in our data base. An example in Fig. 8 shows such a sequence and helps to demonstrate the techniques used to evaluate the parameters of what could be the flapping wave. They are based on the interpretation of vertical plasma velocity $V_{z}$ as a proxy for the up/down translational motions of entire plasma sheet. A test for such a hypothesis could be the anticorrelation between the time derivative $d B_{x} / d t$ and vertical velocity $V_{z}$ (Sergeev et al., 1998). This is definitively the case in the example event, where (see compared time series in the second-top panel) the correlation coefficient between the $d B_{x} / d t$ and $V_{z}$ data points is $C C=0.58$. By integrating $V_{z}$ in time and after detrending the resulting pattern (because of systematic $V_{z}$ offset often existing in the observations of spacecraft rotating around Z-axis) we obtain the estimated vertical motions of the current sheet (top panel). By evaluating the largest difference between subsequent peaks we obtain the apparent amplitude of the vertical wave motion (A, see Fig. 8). 

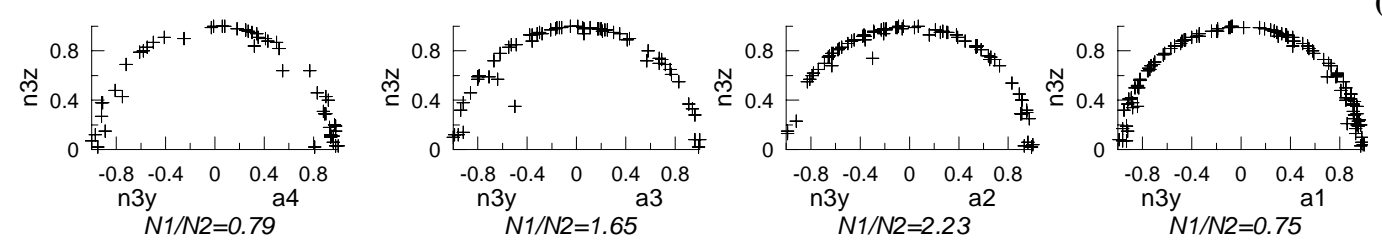

(a)
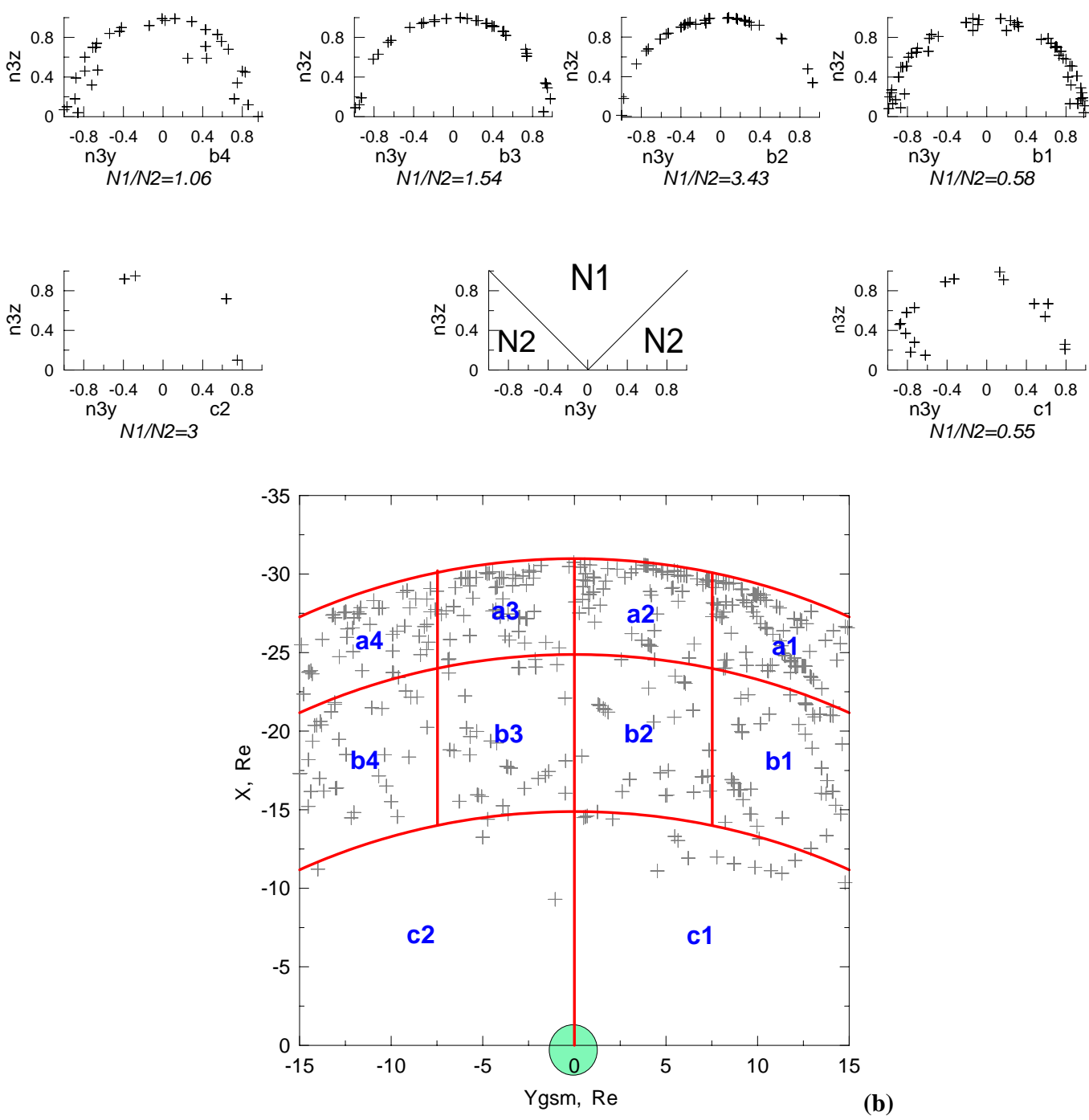

Fig. 7. Distribution of the MVA normals in the YZ plane in the current-sheet related coordinate system. Data are shown separately in different R-Y bins (a) according to the scheme shown in part (b). The ratio of crossings with the normals within the $\pm 45^{\circ}$ cone around the nominal CS sheet normal (N1) and of remaining crossings (N2) is shown to characterise quantitatively the spread of the normals around the $n=1$ circle.

From 51 sequences available, the analysis was done for 26 sequences in which the correlation coefficient between $d B_{x} / d t$ and $-V_{z}$ at a 12-s time resolution was $>0.30$.
Another usage of the $d B_{x} / d t$ and $V_{z}$ correlation is to obtain the estimate of the average apparent vertical sheet half-thickness $\mathrm{H}$ from the regression slope $a$ (from linear fit $\left.d B_{x} / d t=-a V_{z}+b\right)$ and the lobe field $B L$; this gives us $H=B L / a$ (Sergeev et al., 1998). The corresponding statistic is shown in Fig. 9. 


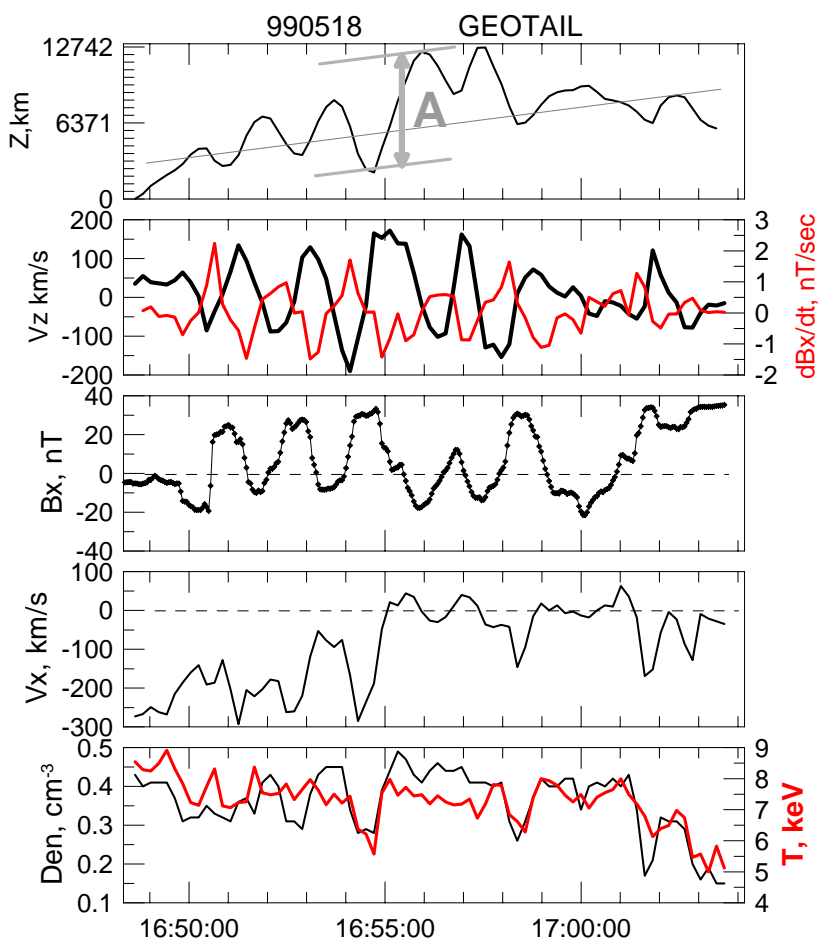

Fig. 8. Example of a sequence of fast current sheet crossings (flapping wave) observed by Geotail on 18 May 1999 at [-16.3; 9.0; 4.5] Re GSM.

The typical amplitude according to this statistic is about $1-2 \mathrm{Re}$, and the sheet thickness is about one Re. The ratio $A / H$ has a median value of about 1 , which implies that the average current sheet thicknesses and amplitudes of its vertical motion are comparable. These values are consistent with previous Cluster analyses made at $\sim 18 \mathrm{Re}$ distance (Runov et al., 2005).

\subsection{Activity dependence}

A large data base of crossings allows us to investigate statistically the relationship of flapping motions with the magnetic activity. Using the preliminary AE index available at 1-min resolution (http://swdcwww.kugi.kyoto-u.ac.jp/aedir/ index.html), we performed a superimposed epoch analysis of $\mathrm{AE}$ behavior during the time interval $\pm 2 \mathrm{~h}$ from the time of the fast crossing, for $\sim 1100$ crossing events in 1997-1999. There are two main lessons from the results shown in Fig. 10. First, many flapping events occur during rather low magnetic activity. For example, the median AE values are modest $(\sim 150 \mathrm{nT})$ and for a quarter of all events there is no significant magnetic activity at all (low quartile is about $50 \mathrm{nT}$ ). The second lesson is that, independent of the activity level, the flapping motions tend to occur at the AE increase phase near the AE maximum, which is usually associated with the substorm expansion phase. Separate analyses made for the
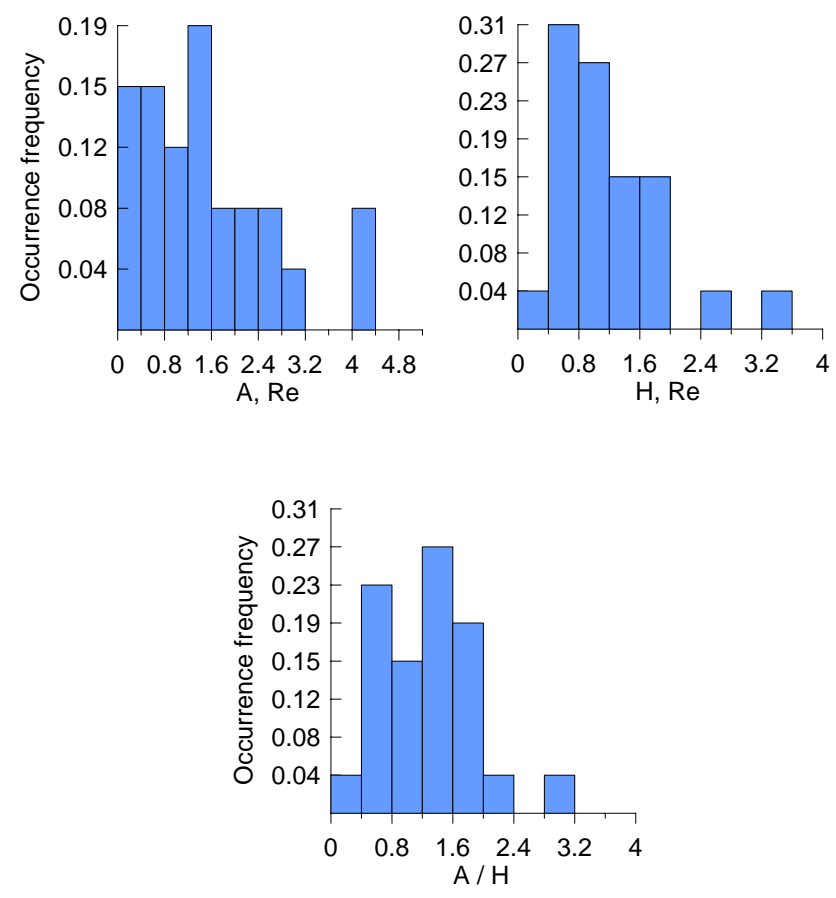

Fig. 9. Distributions of maximal amplitude of the vertical sheet motion and of its apparent thickness, as well as of their ratio in 26 sequences of flapping motions.

subset of events which form the sequences (discussed in previous section) do not show any differences from this general behavior.

\section{Discussion}

Our survey of occurrence and properties of fast current sheet crossings (flapping motions) between 10 to $30 \mathrm{Re}$ in the magnetotail confirmed many results previously obtained by the Cluster system at $r \sim 18$ Re. It concerns such important properties of crossings as the common occurrence of large tilts of the current sheet, and the special orientation of the CS normals (distributed over the circle of $n_{y}^{2}+n_{z}^{2}=1$; see statistics presented in Sergeev et al., 2004 and Runov et al., 2005). Using the additional possibilities of the four-spacecraft observations, these Cluster studies demonstrated that the current sheet surface often display a wavy pattern in subsequent up/down crossings (during sequences similar to those shown in Fig. 8), with alternating tilts of the electric current and related rotations of the CS normal. In rare conjunctions of radially separated Cluster and Double Star spacecraft the synchronous $B_{x}$-oscillatory patterns were reported (Zhang et al., 2005). All this is consistent with a particular "YZ kink"-like mode (see diagram in Fig. 11), which is the most frequent mode of the flapping motions. Our survey of tilt distributions in Fig. 7 expand this conclusion to show that similar properties are observed all along (10-30 Re) and across the midtail. 


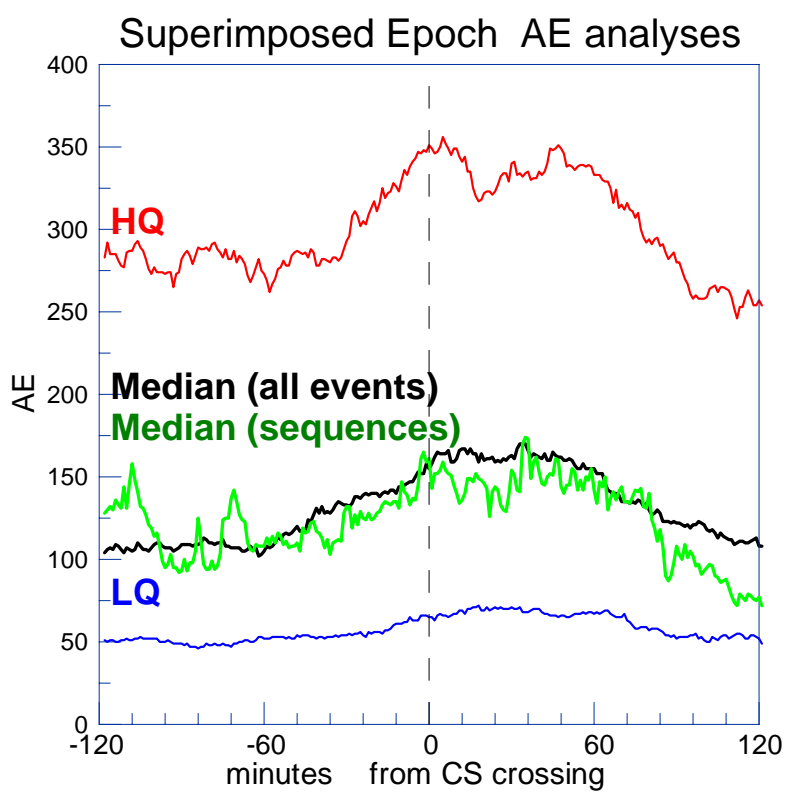

Fig. 10. Superposed epoch analysis of AE-index data with respect to the time of observation of fast CS crossing.

Other average crossing characteristics evaluated in our study (typical crossing duration of the order of $\sim 1 \mathrm{~min}$, wave amplitudes of $\sim 1 \mathrm{Re}$ and CS half thickness about a fraction of $1 \mathrm{Re}$ ) are also similar to those found in Cluster-based surveys (Runov et al., 2005). These major flapping mode characteristics, therefore, do not show considerable changes in the midtail.

Our survey also provides a number of new results. First, the flapping motions are notably more frequent in the central part of the tail than near the flanks (Fig. 5). This is consistent with the source of the flapping motions localized in the tail center, as schematically shown in Fig. 11. That conclusion was previously suggested by the Cluster observation, that in the near-flank tail regions the motions of flapping waves are predominantly from the center to the flanks (Sergeev et al., 2004), as also shown by the arrows in Fig. 11. Both results match each other and confirm an internal origin of the flapping motions, due to some processes localized deep inside of the magnetotail.

The second new result is a strong radial dependence of the flapping occurrence, which, together with a strong dependence of crossing duration on the local flow amplitude (Fig. 6), suggests a hint for the possible source process. The similarity of occurrence patterns in Fig. 5 suggests that either the fast plasma sheet flows (BBFs) are directly related to the generation of flapping motions, or that both phenomena are caused by the same parent process. However, a considerable number of crossings have very weak local flows (see, e.g. second half of crossings in Fig. 8 and statistics in Fig. 5). Therefore, if the BBFs are the process which launches the flapping wave - this wave has to be observed (propagate?)
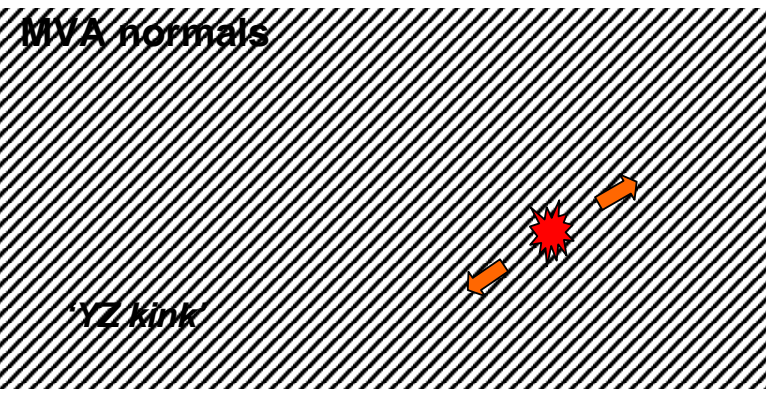

Fig. 11. Scheme showing predicted distributions of MVA normals in the YZ plane for "YZ-kink" type of surface (neutral sheet) waves.

outside of the fast flow stream, or (more probable in our view) both BBFs and the flapping wave could be created by the same parent process, which is the magnetic reconnection. Following Schödel et al. (2001) results we have to emphasize that the sharp decrease in the BBF occurrence with decreasing distance in the midtail does not mean that the flux tube transport is similarly damped, but only that it proceeds with lower velocity (being compensated by the compression of the tubes, so $V \times B z$ is nearly conserved). Therefore, the similarity of BBF and the flapping occurrence implies that either the large speed of the earthward moving plasma, or the proximity to the reconnection region are important conditions to provide the flapping perturbations.

Our statistical result on the preferable occurrence of flapping motions during the AE-index growth (substorm expansion phase) is consistent with previous case studies showing the association of fast crossings with substorm events or disturbed periods (e.g. Toyichi and Miyazaki, 1976; Bauer et al., 1995; Sergeev et al., 1998; etc.), which are also characterized by enhanced magnetic reconnection and BBF activity (Angelopoulos et al., 1994; Nagai et al., 1998; Baumjohann et al., 1999). Observation of some fraction of crossings during very quiet (AE) periods does not contradict this association, since both localized isolated fast streams and magnetic reconnection events are also known to appear under magnetically quiet conditions (Baumjohann et al., 1990; Angelopoulos et al., 1994; Nagai et al., 1998).

As concerns the generation mechanisms, one should first consider the direct mechanism of MHD-type CS deformation during magnetic reconnection. It was suggested that in sheared magnetic configurations (with nonzero $B_{y}$ in the neutral sheet) the interaction of reconnected flux tubes with the sheet is asymmetric and this causes the up-down sheet motions on the flanks of the localized (in Y) reconnection region (Semenov et al., 1994; Shirataka et al., 2006). Predictions of this mechanism regarding the generation of flapping waves are not yet sufficiently explored, but this seems to be the first potential candidate, most directly linked to the generation of the BBFs. A number of other mechanisms were also discussed for kink perturbations, including drift- (e.g. 
Karimabadi et al., 2003) or Kelvin-Helmholtz- (e.g. Nakagawa and Nishida, 1989) instabilities, or a kind of standing mode in the (neutral) current sheet (Fruit et al., 2002a,b). One crucial test is the propagation velocity, which is predicted to be slow and duskward in the case of instabilities. The latter feature conflicts with the flankward propagation direction of the flapping waves, if such propagation is not due merely to the plasma convection (this can be reserved as a possibility which should be further explored). Regarding the propagation effects, the ballooning-like mode in the curved current sheet magnetic field was claimed to be able to propagate azimuthally in flankward directions from the source (Golovchanskaya and Maltsev, 2004). Excitation of nonlinear kink-like waves, with phase velocity an order of magnitude less than the thermal ion speed, was reported in numerical kinetic simulations of initially very thin current sheets (Karimabadi et al., 2003) and of bifurcated sheets with realistic thickness (Sitnov et al., 2004), which give the perturbation shape and scales similar to some of observed events. More work has to be done to clarify all of these issues.

Acknowledgements. The preliminary AE index data were made available at Kyoto WDC-C data base and Geotail data are available at DARTS website. We thank N. Dmitrieva and S. Dubyagin for their help in data analysis and discussions. We also thank L. Zelenyi, A. Petrukovich and M. Sitnov for fruitful discussions. VAS and SVA thanks Austrian Academy of Science for the support during their stays in Graz. This work was supported by INTAS grant 03-51-3738, RFBR grant 03-05-20012, WTZA AustriaRussia grant N I.1/04 as well as Russian Ministry of science grant 15392.

Topical Editor I. A. Daglis thanks two referees for their help in evaluating this paper.

\section{References}

Angelopoulos, V., Kennel, C. F., Coroniti, F. V., et al.: Statistical characteristics of bursty bulk flow events. J. Geophys. Res., 99, $21257-21280,1994$.

Bauer, T. M., Baumjohann, W. and Treumann, R. A.: Neutral sheet oscillations at substorm onset, J. Geophys. Res., 100, $23737-$ $23742,1995$.

Baumjohann, W., Paschmann,G., and Lühr, H.: Characteristics of high-speed ion flows in the plasma sheet. J. Geophys. Res., 95, 3801-3810, 1990.

Chanteur, G.: Spatial interpolation for four spacecraft: Theory, in Analysis Methods for Multi-Spacecraft Data, edited by: Paschmann, G. and Daly, P., ESA, Noordwijk, 349-369, 1998.

Fairfield, D. H., Hones Jr, E. W., and Meng, C.-I.: Multiple crossing of a very thin plasma sheet in the Earth's magnetotail, J. Geophys. Res., 86, 11 189-11 200, 1981.

Fruit, G., Louarn, P., Tur, A., and Le Quéau, D.: Propagation of MHD perturbations in a Harris current sheet. 1- discrete modes, J. Geophys. Res., 107, 1411, doi:10.1029/2001JA009212, 2002a.

Fruit, G., Louarn, P., Tur, A. and Le Quéau, D.: Propagation of MHD perturbations in a Harris current sheet. 2 - continuous spectrum and resonant absorption, J. Geophys. Res., 107, 1412, doi:10.1029/2001JA009215, 2002b.

Golovchanskaya, I. V. and Maltsev, Yu. P.: On the identification of plasma sheet flapping waves observed by Cluster, Geophys. Res. Lett., 32, L02102, doi:10.1029/2004GL021552, 2004.

Halland, S. E., Sonnerup, B., Dunlop, M. W., Balogh, A.,Georgescu, E., et al.: Four-spacecraft determination of magnetopause orientation, motion and thickness: comparison with results from single-spacecraft methods, Ann. Geophys., 22, 1347-1365, 2004.

Karimabadi, H., Pritchett, P. L., Daughton, W., and KraussVarban, D.: Ion-ion kink instability in the magnetotail: 2 Three-dimensional full particle and hybrid simulations and comparison with observations, J. Geophys. Res., 108, 1401, doi:10.1029/2003JA010109, 2003.

Knetter T., Neubauer, F. M., Horbury, T., and Balogh, A.: Fourpoint discontinuity observations using Cluster magnetic field data: A statistical survey, J.Geophys. Res., 109, A061-2, doi:10.1029/2003JA010099, 2004.

Lui, A. T. Y, Meng, C.-I., and Akasofu, S.-I.: Wavy nature of the magnetotail neutral sheet, Geophys. Res. Lett., 5, 279-282, 1978.

McComas, D. J., Russel, C. T., Elphic, R. C., and Bame, S. J.: The near-Earth cross-tail current sheet: Detailed ISEE 1 and 2 case studies, J. Geophys. Res., 91, 4287-4301, 1986.

Nagai, T., Fujimoto, M., Saito, Y., et al : Structure and dynamics of magnetic reconnection for substorm onsets with Geotail observations, J. Geophys. Res., 103, A3, 4419-4440, 1998.

Nakagawa, T. and Nishida, A.: Southward magnetic field in the neutral sheet produced by wavy motions in the dawn-dusk direction, Geophys. Res. Lett., 11, 1265-1268, 1989.

Runov, A., Sergeev, V. A., Baumjohann, W., Nakamura, R., Apatenkov, S., et al : Electric current and magnetic field geometry in flapping magnetotail current sheets, Ann. Geophys, 23, 13911403, 2005.

Schödel, R., Baumjohann, W., Nakamura,R., Sergeev, V. A., and Mukai, T.: Rapid flux transfer in the central plasma sheet, J. Geophys. Res., 106, 301-313, 2001.

Semenov, V. S., Farrugia, C. J., Biernat, H. K., et al.: Reconnectionassociated surface waves at the magnetopause, Geophys. Res. Lett., 21, 2437-2440, 1994.

Sergeev, V., Angelopulous, V., Carlson, C., and Sutcliffe, P.: Current sheet measurements within a flapping plasma sheet, J. Geophys. Res, 103, 9177-9188, 1998.

Sergeev, V., Runov, A., Baumjohann, W., Nakamura, R., Zhang, T. L., et al: Orientation and propagation of current sheet oscillations, Geophys. Res. Lett., 31, L05 807, doi:10.1029/2003GL019346, 2004.

Shirataka, N., Fujimoto, M., Hasegawa, H., and TanDokoro, R.: Reproducing the bi-polar magnetic signature at the jet leading edge by three-dimensional reconnection with non-zero guide field, J. Geophys. Res., 111, A07201, doi:10.1029/2005JA011521, 2006.

Sitnov, M. I., Swisdak, M., Drake, J. F., Guzdar, P. N., and Rogers, B. N.: A model of the bifurcated current sheet: 2. Flapping motion, Geophys. Res. Lett., 31, L09 805, doi:10.1029/2004GL019473, 2004.

Sonnerup, B. U. and Schneible, M.: Minimum and maximum variance analysis, Analysis Methods for Multi-Spacecraft Data, edited by: Paschmann, G. and Daly, P., ISSI Scientific Report 
SR-001, ISSI/ESA, 185-220, 1998.

Speiser, T. W. and Ness, N. F.: The neutral sheet in the geomagnetic tail: Its motion, equivalent currents, and field line reconnection through it, J. Geophys. Res., 72, 131-141, 1967.

Toyichi, T. and Miyazaki, T.: Flapping motions of the tail plasma sheet induced by the interplanetary magnetic field variations, Planet. Space Sci., 24, 147-156, 1976.

Tsyganenko, N. A. and Fairfield, D. H. : Global shape of the magnetotail current sheet as derived from Geotail and Polar data, J. Geophys. Res., 109, A03218, doi:10.1029/2003JA010062, 2004.

Volwerk, M., Glassmeier, K.-H., Runov, A., Nakamura, R., Baumjohann, W., et al. : Flow burst induced large scale plasma sheet oscillation, J. Geophys. Res., 109, A11208, doi:10.1029/2004JA010533, 2004.
Volwerk, M., Glassmeier, K.-H., Runov, A., Baumjohann, W., Nakamura, R., et al.: Kink mode oscillation of the current sheet, Geophys. Res. Lett., 30, 1320, doi:10.1029/2002GL016467, 2003.

Zhang, T. I., Baumjohann, W., Nakamura, R., Balogh, A., and Glassmeier, K.-H. : A wavy twisted neutral sheet observed by Cluster, Geophys. Res. Lett., 29, 1899, doi:10.1029/2002GL015544, 2002.

Zhang, T. L., Nakamura, R., Volwerk, M., Runov, A., Baumjohann, W., et al.: Double Star/Cluster observation of neutral sheet oscillations on August 5, 2004, Ann. Geophys., 2909-2914, 2005. 